Este obra forma parte del acervo de la Biblioteca Jurídica Virtual del Instituto de Investigaciones Jurídicas de la UNAM

\title{
¿ALGO NUEVO DESDE EL FIN DE LA GUERRA FRÍA? O EL IMPACTO DEL DERECHO INTERNACIONAL EN EL DERECHO NACIONAL: NORMAS ELECTORALES INTERNACIONALES Y SU LEGITIMIDAD
}

Christina BINDER ${ }^{1}$

\section{Sumario:}
I. Introducción
II. Normas electorales internacionales post-1989
III. Perspectivas de legitimidad de las normas electorales internacionales: una comparación
IV. Consideraciones finales

\section{INTRODUCCIÓN}

Tras el final de la guerra fría y el colapso de la Unión Soviética el espíritu democrático del tiempo, el Zeitgeist, se extendió por el mundo. ${ }^{2}$ Por un lado, el derecho internacional ya no luce indiferente a la manera en cómo se constituye un régimen político y por el otro, se hace cada vez más perceptible el surgimiento de un derecho democrático. Estas dos constelaciones se mueven junto a un mayor desarrollo y aplicación de las normas electorales internacionales.

\footnotetext{
${ }^{1}$ Profesora en el Departamento de Derecho europeo, internacional y comparado de la Universidad de Viena; ha sido becaria de la Academia de Ciencias de Austria; investigador visitante en el Centro Lauterpacht de Derecho internacional en Cambridge en el periodo (2007-2008), investigador visitante en el Instituto MaxPlanck de Derecho Público Comparado y Derecho Internacional en Heidelberg. La autora desea agradecer a Ursula Kriebaum y a la Dienstagsrunde, especialmente al doctor Armin von Bogdandy, Jochen von Bernstorff, Isabel Feichtner y Matthias Goldmann por los valiosos comentarios sobre una versión anterior de este documento, así como a Nils Meyer-Ohlendorf y Philipp Dann, por sus aportes en los debates.

2 Diamond, L., Plattner, M. et al., Consolidating the Third Wave Democracies. Themes and Perspectives, 1997.
} 
Este obra forma parte del acervo de la Biblioteca Jurídica Virtual del Instituto de Investigaciones Jurídicas de la UNAM www.juridicas.unam.mx

El fenómeno del impacto del "derecho internacional en el derecho nacional" en materia electoral posterior a 1989, corresponde a una creciente expansión del derecho internacional hacia el derecho interno de los Estados, fenómeno que igualmente ha tenido lugar en otros ámbitos, como el derecho ambiental y económico. ${ }^{3}$ Un aspecto de esa expansión en el ámbito nacional, es que el derecho internacional se ocupa cada vez más no sólo de los Estados, sino también de los actores no estatales. ${ }^{4}$ Esto viene aparejado por una mayor presencia y desarrollo de instituciones internacionales ${ }^{5}$ que cuentan con amplias competencias y vida propia.

Estos desarrollos implican nuevos desafíos para justificar el estado del derecho internacional. En términos de legitimidad —entendida como "una cualidad que permite a las personas o Estados aceptar autoridad- independiente de la coerción, el interés propio, o la persuasión racional, a causa de una sensación general de que la autoridad está justificada" ${ }^{6}$ se plantea la pregunta vinculada al fundamento sobre cómo el funcionamiento del derecho internacional es aceptado por sus destinatarios.

${ }^{3}$ Véase Bodansky, D., “The Legitimacy of International Governance: A Coming Challenge for International Environmental Law" 93, American Journal of International Law, 1999, p. 596; Wolfrum, R., "Legitimacy of International Law from a Legal Perspective: Some Introductory Considerations" en Wolfrum, R. y Röben, V. (eds.), Legitimacy of International Law, 2008, pp. 1 y 12; Weiler, J.H.H., "The Geology of International LawGovernance, Democracy and Legitimacy", 64 Zeitschrift für ausländisches öffentliches Recht und Völkerrecht, 2004, p. 547; Slaughter, A.M., Burke-White, W., “The Future of International Law is Domestic. (Or $<<$ The European Way of Law>>)", 47 Harvard International Law Journal, 2006, p. 327.

${ }^{4}$ Friedmann, W., The Changing Structure of International Law, 1964, pp.60 y ss; Tomuschat, C., "International Law: Ensuring the Survival of Mankind on the Eve of a New Century", 281 Recueil des Cours, 2001, pp. 13, y 70; Kumm, M., "The Legitimacy of International Law: A Constitutionalist Framework of Analysis", 15 European Journal of International Law, 2004, p. 907.

${ }^{5}$ Para una definición del ejercicio de la autoridad pública internacional, véase Von Bogdandy, A., Dann, P. et al., "Developing the Publicness of Public International Law: Towards a Legal Framework for Global Governance Activities", en Von Bogdandy, A., Wolfrum, R. et al., (eds.), The Exercise of Public Authority by International Institutions. Advancing International Institutional Law, 2009, pp. 3, 11 y ss.

${ }^{6}$ Véase Bodansky, supra nota, p.600. Sobre similares puntos de vista de la legitimidad como la justificación de la autoridad, véase Wolfrum, supra nota; Bodansky, D., "Legitimacy" en Hey, E., Brunnée, J. et al. (eds.) Oxford Handbook of International Environmental Law, 2007, p.704; Delbrück, J., “Exercising Public Authority beyond the State: Transnational Democracy and/or Alternative Legitimation Strategies?,"10 Indiana Journal of Global Legal Studies, 2003, p. 29. 
Este obra forma parte del acervo de la Biblioteca Jurídica Virtual del Instituto de Investigaciones Jurídicas de la UNAM www.juridicas.unam.mx

En el derecho internacional tradicional o sistema de Westfalia, el consentimiento del Estado y la legalidad fueron considerados, en gran medida, como elementos suficientes para aceptar las normas del derecho internacional; éste fue percibido tradicionalmente como ley entre los Estados. Los Estados soberanos se vinculaban o consentían mutuamente las obligaciones internacionales. ${ }^{7}$ En la medida en que los Estados se sometían a la autoridad de las instituciones internacionales, la legalidad desempeñó un papel de legitimación al conectar continuamente la autoridad de las instituciones, al consentimiento estatal, dado en el documento constitutivo como su base originaria. ${ }^{8} \mathrm{Sin}$ embargo, esas tradicionales justificaciones parecen cada vez más insuficientes en vista del fenómeno del "impacto del derecho internacional en el derecho nacional" con diferentes destinatarios; como por ejemplo, los actores no estatales que han sido admitidos y un papel cada vez más activo de las instituciones internacionales. ${ }^{9}$

La insuficiencia de consentimiento y legalidad estatal caracterizan el desarrollo y la aplicación de las normas electorales internacionales por parte del órgano internacional de monitoreo o vigilancia de los derechos humanos. Entre dichos órganos hay que destacar particularmente los tribunales regionales, como el Tribunal Europeo de Derechos Humanos (en adelante TEDH) y la Corte Interamericana de Derechos Humanos, ${ }^{10}$ así como las organizaciones internacionales que realizan misiones de observación electoral. Su dinamismo difícilmente establece con solidez la acción de las instituciones internacionales con el consentimiento original firmemente concedido por el Estado. Esto parece particularmente sensible, ya que ambas instituciones tocan el

${ }^{7}$ La separación entre el derecho interno e internacional, como afirma Krisch-Kingsbury, ha sido durante mucho tiempo un medio para limitar las demandas de legitimidad en el derecho internacional, véase Krisch, N. y Kingsbury, B., "Introduction: Global Governance and Global Administrative Law in the International Legal Order," 17 European Journal of International Law, 2006, pp. 1 y 11; Kingsbury, B., "Sovereignty and Inequality", 9 European Journal of International Law, 1998, 599.

${ }^{8}$ Véase Bodansky, supra nota, p.712.

${ }^{9}$ Ibidem, pp.596 y ss., 610. Cfr. Wolfrum, R., "Legitimacy of International Law and the Exercise of Administrative Functions: The Example of the International Seabed Authority, the International Maritime Organization (IMO) and International Fisheries Organizations", 9 German Law Journal, 2008, pp. 2041 y 2044.

${ }^{10}$ Véase Int.-American Court of Human Rights, Yatama vs. Nicaragua, Judgment, 23 de junio de 2005, serie C, núm. 127; Aguiar, A., El derecho a la democracia. La democracia en el derecho y la jurisprudencia interamericanos. La libertad de expresión, piedra angular de la democracia, 2008, pp.125, 355 y ss. 
Este obra forma parte del acervo de la Biblioteca Jurídica Virtual del Instituto de Investigaciones Jurídicas de la UNAM www.juridicas.unam. $\mathrm{mx}$

corazón mismo del funcionamiento de un Estado, con un potencial impacto de largo alcance: el marco electoral en su orden constitucional.

Por lo tanto, hacen falta otras maneras de explicar y evaluar el ejercicio de su autoridad. Dicho de otra manera, parece necesario examinar si el desarrollo y aplicación de las normas electorales internacionales pueden ser consideradas legítimas por razones distintas a las fundamentadas en el consentimiento del Estado y en la legalidad. De hecho, además de la legitimidad derivada del consentimiento del Estado como fuente originaria, otras dimensiones de legitimidad han sido propuestas en la literatura para justificar el ejercicio de la autoridad internacional, entre las que se encuentran: los aspectos de legitimidad procesal y los aspectos sustantivos orientados a los resultados. ${ }^{11}$

En ese contexto, este artículo se propone examinar y comparar el desarrollo, y la aplicación de las normas electorales internacionales en las dos líneas jurídicas ${ }^{12}$ de esas perspectivas de legitimidad adicionales. ${ }^{13}$ Tal investigación parece prometedora dado el desarrollo paralelo y en gran medida no relacionado con las normas de derecho internacional de los derechos humanos y la práctica de la observación electoral de las organizaciones internacionales, convirtiéndolos en buenos casos para demostrar que "el derecho internacional ha penetrado al derecho nacional" en el mismo campo pero por diferentes medios.

En consecuencia, iniciaremos con una breve reseña sobre el surgimiento del derecho a la democracia como una prerrogativa del derecho internacional. Luego se hará una evaluación de las normas electorales en el contexto regional europeo, mediante el examen de la jurisprudencia del TEDH y la práctica de observación electoral de la Oficina de Instituciones Democráticas y Derechos Humanos (OIDDH) de la Organización para la

\footnotetext{
${ }^{11}$ Véase Bodansky, supra nota; Wolfrum, supra nota; Bodansky, supra nota; Treves, T., "Aspects of Legitimacy of Decisions of International Courts and Tribunals", en Wolfrum, R. y Röben, V. (eds.), Legitimacy in International Law, 2008, p.169. No solamente sobre la perspectiva del derecho internacional de la legitimidad, véase Bernstein, S., "Legitimacy in Global Environmental Governance", 1 Journal of International Law and International Relations, 2004-2005, p.139.

12 "Norma” se define ampliamente, incluyendo también las normas no vinculantes.

${ }^{13}$ Escapa al alcance de este documento, examinar nuevas estrategias de legitimación discutidas en la literatura, conceptos tales como democracia transnacional. Para mayor referencia cfr. Delbrück, supra nota, pp. 5, 34 y ss; Kumm, supra nota, p.3.
} 
Este obra forma parte del acervo de la Biblioteca Jurídica Virtual del Instituto de Investigaciones Jurídicas de la UNAM www.juridicas.unam.mx

DOl: http://dx.doi.org/10.22201/iij.24487910e.2015.7.10083

Seguridad y la Cooperación en Europa (OSCE). ${ }^{14}$ Se argumentará, que el consentimiento del Estado, mediante la ratificación del Convenio Europeo de Derechos Humanos (CEDH) y la invitación de las misiones de observación electoral en el Documento de Copenhague de 1990, es una base muy débil para el desarrollo y la aplicación de las normas electorales internacionales por parte del TEDH y la OIDDH, especialmente teniendo en cuenta el impacto potencial de sus hallazgos (Segunda parte). Es por eso que en la tercera parte se utilizaron tres dimensiones de legitimidad y de procedimiento relacionadas con la obtención de resultados adicionales para evaluar y comparar el desarrollo y la aplicación de las normas electorales internacionales en las dos vertientes de la ley, y en la cuarta parte presentaremos unas reflexiones a modo de conclusiones.

\section{LAS NORMAS ELECTORALES INTERNACIONALES \\ POST-1989}

El desarrollo de las normas electorales internacionales tras el fin de la guerra fría tuvo lugar en el contexto de una creciente preocupación internacional por la gobernanza democrática en el nivel nacional. En la presente sección, primero se ofrecerá una visión general de los cambios en relación con el derecho a la democracia en el derecho internacional post 1989 (2.1.) y segundo se examinará el desarrollo y aplicación de las normas electorales internacionales en Europa (2.2.).

\section{Contexto normativo: el derecho a la democracia}

en el derecho internacional

El derecho emergente a la democracia en el derecho internacional consuetudinario es un fenómeno reciente. Durante muchos años, el derecho internacional se percibe estrictamente neutral hacia los ordenamientos constitucionales nacionales, especialmente relacionado a cómo se forman los gobiernos nacionales. ${ }^{15}$ En 1986, en su famosa decisión sobre Nicaragua, la Corte Internacional de Justicia todavía parecía reafirmar la "ceguera" del derecho internacional hacia la estructura interna del poder del Estado, es decir, la forma de gobierno en un Estado determinado, al declarar que: "sea

\footnotetext{
${ }^{14}$ La región de la OSCE excede geográficamente Europa, ya que sus 57 Estados miembros comprenden también los Estados de América del Norte y Asia Central.

${ }^{15}$ Los primeros intentos de introducir la noción de "legitimidad" como criterio para el reconocimiento de los gobiernos en virtud del derecho internacional incluyen, la doctrina Tobar enunciada en América Latina en 1907. Aún así, no tuvo un efecto duradero en la práctica internacional.
} 
Este obra forma parte del acervo de la Biblioteca Jurídica Virtual del Instituto de Investigaciones Jurídicas de la UNAM www.juridicas.unam.mx

cual fuere la definición que se haga del régimen de Nicaragua, ${ }^{16}$ la adhesión de un Estado a cualquier doctrina particular no constituye una violación del derecho internacional consuetudinario". ${ }^{17}$ Como subrayó James Crawford, "la reacción negativa de la Corte, incluso a la idea de que Nicaragua estuviera sujeta a la supervisión internacional o a la rendición de cuentas en la realización de las elecciones, refleja el énfasis del derecho internacional tradicional de no intervención en los asuntos internos de los Estados".

La mayoría de los instrumentos de derechos humanos enmarcan vagamente el derecho a la participación política, tanto a nivel global como regional, utilizando términos como "elecciones periódicas auténticas" o "que garanticen la libre expresión de la voluntad de los electores". ${ }^{18}$ Las disposiciones relativas a los derechos políticos no fueron seriamente adjudicadas hasta que se produjo la ola de democratizaciones después del final de la guerra fría, ${ }^{19}$ es decir, que excepcionalmente ocurrió antes de 1989. El Tribunal Europeo de Derechos Humanos, por ejemplo, no emitió sino hasta 1987 su primera sentencia sobre el derecho a elecciones libres. ${ }^{20}$ Del mismo modo, hubo poca observación electoral o monitoreo antes de 1989, y fue principalmente en el proceso de descolonización que la ONU supervisó plebiscitos, referéndums de independencia y elecciones aproximadamente en 30 territorios no autónomos entre 1956 y $1989 .{ }^{21}$

${ }^{16}$ Véase, caso Concerning Military and Paramilitary Activities in and against Nicaragua (Nicaragua vs. United States, Judgment, 27 de junio de 1986, ICJ Reps, 1986, pp. 98 y 133.

${ }^{17}$ Crawford, J., “Democracy and International Law', 64 British Yearbook of International Law”, 1993, pp.113 y 121; Pippan, C., "International Law, Domestic Political Orders and the <<Democratic Imperative>>: Has Democracy Finally Emerged as a Global Legal Entitlement?", Jean Monnet Working Paper No. 2/10, Nueva York, 2010, http://centers.law.nyu.edu/jeanmonnet/papers/papers10.htm/

${ }^{18}$ Artículo 25 CCPR, 1966; véanse normas similares en los siguientes artículos: 23 ACHR, 1969; 13 Banjul Charter, 1981 y el marco legal impreciso para elecciones libres en el artículo 3o., del primer protocolo del CEDH, 1952. En la indeterminación general de los derechos políticos, véase Nowak, M., Un Covenant on Civil and Political Rights. CCPR Commentary, 2da. ed., 2005, pp. 562 y 590; Fox, G., "The Right to Political Participation in International Law", en Fox, G. y Roth, B. (eds.), Democratic Governance and International Law, 2000, p. 48; Steiner, H., “Political Participation as a Human Right”, Harvard Human Rights Yearbook, 1988, pp. 77 y 131.

${ }^{19}$ Véase Fox, supra nota, p. 69.

${ }^{20}$ ECtHR, Mathieu-Mohin and Clerfayt vs. Belgium, 2 de marzo de 1987, A 113, 1987.

${ }^{21}$ Véase Binder, C. y Pippan, C., “Election Monitoring, International”, en Wolfrum, R., (ed.), Encyclopedia of 
Este obra forma parte del acervo de la Biblioteca Jurídica Virtual del Instituto de Investigaciones Jurídicas de la UNAM www.juridicas.unam.mx

DOl: http://dx.doi.org/10.22201/iij.24487910e.2015.7.10083

Esta falta de preocupación internacional por los procesos electorales nacionales cambió drásticamente después de la guerra fría. Los Estados sucesores de la Unión Soviética, especialmente los de Europa Central y Oriental, eligieron en gran medida la democracia como su forma de gobierno. En América Latina, los regímenes democráticamente electos reemplazaron las dictaduras militares y en varios países de África se llevaron a cabo reformas democráticas. La práctica estatal reciente, así como la legislación y la práctica de las organizaciones internacionales indican que el derecho internacional ya no es indiferente a aquellos regímenes que invocan la soberanía para ejercer un mayor control dentro de las fronteras nacionales. ${ }^{22}$

Desde la perspectiva del derecho internacional, Thomas Franck ya se refirió en 1992 a un "emergente derecho a la gobernanza democrática". ${ }^{23}$ Niels Petersen en 2008, reformuló ligeramente la propuesta de Franck e identificó un "derecho al establecimiento de la gobernanza democrática". ${ }^{24}$ Por lo tanto, Petersen desarrolló un principio de la teleología democrática, según el cual los Estados estarían obligados a evolucionar hacia la democracia, la consolidación de las instituciones democráticas y evitar regresiones en el proceso de democratización. ${ }^{25}$

Estos estudios sobre el establecimiento del derecho a la gobernanza democrática evidencian una tendencia cada vez mayor de que el "derecho internacional va al derecho nacional" en términos de gobernanza nacional ${ }^{26}$ posterior a 1989 y representa el fondo

Public International Law, 2da. ed., 2008, http://WwW.mpepil.com/, párrafo 8.

${ }^{22}$ Fox, G. y Roth, B., “Introduction: the Spread of Liberal Democracy and its Implications for International Law", en Fox, G. y Roth, B. (eds.), Democratic Governance and International Law, 2000, pp. 1 y 2.

${ }^{23}$ Franck, T., “The Emerging Right to Democratic Governance”, 86 American Journal of International Law, 1992, p. 46.

${ }^{24}$ Petersen, N., "The Principle of Democratic Teleology in International Law", 34 Brooklyn Journal of International Law, 2008, pp. 33 y 84; d'Asprémont, J., L'État Non Démocratique en Droit International. Étude Critique du Droit International Positif et de la Pratique Contemporaine, 2008; Pippan, C., "Gibt es ein Recht auf Demokratie im Völkerrecht?", en Riefler, R. (ed.), Popper und die Menschenrechte, 2007, p.119. Para el contexto regional europeo, véase Wheatley, S., “Democracy in International Law: a European Perspective, " 51 International and Comparative Law Quarterly, 2002, p. 225.

${ }^{25}$ Petersen, N., Demokratie als teleologisches Prinzip. Zur Legitimität von Staatsgewalt im Völkerrecht , 2009, pp. 2, 21, 49 y ss. El principio, de acuerdo con Petersen, sufre indeterminación y se orientada a cada proceso.

${ }^{26}$ Para los críticos de esta prerrogativa véanse Schindler, D., "Völkerrecht und Demokratie", en Hafner, G. et 
Este obra forma parte del acervo de la Biblioteca Jurídica Virtual del Instituto de Investigaciones Jurídicas de la UNAM www.juridicas.unam.mx

normativo de este análisis. Aún así, el desarrollo y la aplicación de las normas electorales internacionales no son directamente dependientes de la existencia de tal derecho, ya que se sustentan en la participación de los Estados en los tratados de derechos humanos y la invitación de las misiones de observación electoral. ${ }^{27}$ Sobre esta base, vamos a proceder a examinar cómo "el derecho internacional ha avanzado al ámbito nacional" en el contexto regional de Europa, con especial atención a la jurisprudencia del TEDH y la práctica de observación electoral de la OIDDH.

\section{Desarrollo y aplicación en Europa de las normas}

\section{electorales internacionales}

El TEDH en su interpretación del derecho a elecciones libres y la OIDDH han adoptado una postura cada vez más proactiva en materia electoral desde el fin de la guerra fría. A pesar de los diferentes enfoques del TEDH en la protección de los derechos individuales y la evaluación general del proceso electoral que corresponde a las misiones de observación electoral de la OIDDH, ambas instituciones han contribuido en la construcción y aplicación de normas electorales. En primer lugar, tal desarrollo se evidencia en la evolución de la jurisprudencia del TEDH en el ámbito de aplicación del artículo 3o. del primer Protocolo (P) del CEDH, en los casos que han sido presentados ante el TEDH desde su primera decisión en $1987 .{ }^{28}$ Del mismo modo, las misiones de observación electoral desplegadas hasta ahora, ${ }^{29}$ han aplicado y concretado los compromisos electorales contenidos en el Documento de Copenhague de $1990,{ }^{30}$

al. (eds.), Liber Amicorum Professor Seidl-Hohenveldern, 1998, p. 611; Bauer, B., Der völkerrechtliche Anspruch auf Demokratie, 1998; Carothers, T., "Empirical Perspectives on the Emerging Norm of Democratic Governance", ASIL Proceedings, 1992, p.261; Koskenniemi, M., "Whose Intolerance, which Democracy?" en Fox, G. y Roth, B. (eds.), Democratic Governance and International Law, 2000, p. 436.

${ }^{27}$ Véase Petersen, N., supra nota, p.106.

${ }^{28}$ En total, son 40 casos entre 1987 y 2009; Harris, D.J., O'Boyle, M. et al., Harris, O'Boyle \& Warbrick. Law of the European Convention on Human Rights, 2da. ed., 2009, p. 711; Golubok, S., "Right to Free Elections: Emerging Guarantees or Two Layers of Protection?," 27 Netherlands Quarterly of Human Rights, 2009, p. 361.

${ }^{29}$ Son más de 230 misiones hasta 2010, OSCE-ODIHR, Election Observation Handbook, 6ta. ed., 2010, p. 8. Mientras que los formatos de las misiones de observación electoral difieren, la siguiente evaluación se basará en una misión "estandarizada-normalizada”.

${ }^{30}$ Document of the Copenhagen Meeting of the Conference on the Human Dimension of the CSCE, 29 de junio de 1990, http://www.osce.org/documents/odihr/1990/06/13992_en.pdf. 
Este obra forma parte del acervo de la Biblioteca Jurídica Virtual del Instituto de Investigaciones Jurídicas de la UNAM www.juridicas.unam.mx

DOl: http://dx_doi.org/10.22201/ijj.24487910e_2015.7.10083

generando con ello soft law y las mejores prácticas. En segundo lugar, ambas instituciones han promovido el cumplimiento y la aplicación de las normas electorales. Por un lado, el TEDH a través de sus decisiones vinculantes sobre derechos políticos cuya ejecución es supervisada por el Comité de Ministros, y por el otro, las misiones de observación electoral de la OIDDH a través de las presiones políticas ejercidas sobre los Estados para que se realicen los procesos electorales, de conformidad con el derecho internacional, así como a través de la publicación de sus observaciones y el posterior seguimiento de ellas.

También, tal como se argumentará seguidamente, ambas instituciones se basan fundamentalmente en las competencias atribuidas en los documentos fundacionales (CEDH y sus Protocolos; documentos de la OSCE) y en la propia dinámica de desarrollo y aplicación de las normas electorales.

\section{A. La jurisprudencia del Tribunal Europeo}

de Derechos Humanos (TEDH)

Sustentar la jurisprudencia evolutiva del TEDH sobre el derecho a elecciones libres en el artículo 3o. del primer Protocolo (P) del CEDH, ha sido un proceso obstaculizado desde un principio por su imprecisa redacción que refleja de alguna manera, la polémica con respecto a la incorporación de los derechos políticos en el Convenio. ${ }^{31}$ De hecho, algunos expertos cuestionaron durante la redacción del Convenio, si deberían incluirse en el CEDH "cuestiones de carácter constitucional y político". Al final, el derecho a la libre elección fue consagrado en el artículo 30. del primer Protocolo (P) del CEDH y posteriormente adoptado en $1952 .{ }^{32}$ Sin embargo, la redacción imprecisa de dicho artículo es notaria: ${ }^{33}$ en lugar de conceder derechos individuales, el artículo 3o. se limita

\footnotetext{
${ }^{31}$ Véase Marks, S., "The European Convention on Human Rights and its "Democratic Society", 56 British Yearbook of International Law, 1995, pp. 209 y 221.

32213 UNTS 262; ETS 9; adoptado en 1952, entró en vigencia en 1954; hoy cuenta con 47 participantes. El primer Protocolo del CEDH fue ratificado por todos los Estados miembros del Consejo de Europa, con la excepción de Suiza y Mónaco. Estatus de ratificaciones a partir de junio de 2015, http://conventions.coe.int/Treaty/Commun/ChercheSig.asp?NT=009\&CM=7\&DF=11/09/2011\&CL=ENG.

${ }^{33}$ Los trabajos preparatorios no son suficientemente claros en cuanto a si los Estados tienen la intención de crear un derecho exigible por individuos para contar con elecciones libres o más bien estipular la obligación general -no exigibles por individuos - a los Estados de mantener las estructuras democráticas, véase Marks, supra nota, p.221.
} 
Este obra forma parte del acervo de la Biblioteca Jurídica Virtual del Instituto de Investigaciones Jurídicas de la UNAM www.juridicas.unam.mx

DOI: http://dx.doi.org/10.22201/iij.24487910e_2015.7.10083

a establecer obligaciones para los Estados, en el sentido de celebrar en intervalos razonables, elecciones libres y secretas. ${ }^{34}$

La jurisprudencia temprana del TEDH en materia de derechos políticos confirma esta debilidad del artículo 3o. antes mencionado. EI TEDH ha ejercido considerable autocontrol, dejando un amplio margen de apreciación a los Estados y se limita a controlar si la esencia del derecho fue violado en un esquema de comprobación, para determinar si la interferencia del Estado fue desproporcionada o arbitraria. ${ }^{35}$ No obstante, en su más reciente jurisprudencia, el TEDH ha adoptado un enfoque más afirmativo, ${ }^{36}$ sometiendo los marcos electorales de los respectivos Estados a un escrutinio más estricto. En muchos de los casos relacionados con el derecho a presentarse a las elecciones, el Tribunal adoptó una evaluación de proporcionalidad más escrupulosa, sopesando los aspectos individuales, con los intereses del respectivo Estado. $^{37}$

En su interpretación evolutiva del artículo 3o. del Protocolo núm.1 del CEDH, el TEDH amplió considerablemente su alcance. ${ }^{38}$ El TEDH razonó el desarrollo de las normas electorales mediante la comparación con los sistemas jurídicos de los Estados miembros

\footnotetext{
${ }^{34}$ Artículo 3o., primer Protocolo: “las Altas Partes Contratantes se comprometen a organizar, a intervalos razonables, elecciones libres con escrutinio secreto, en condiciones que garanticen la libre expresión de la opinión del pueblo en la elección del cuerpo legislativo..."

${ }^{35}$ Mathieu-Mohin and Clerfayt vs. Belgium, supra nota, párrafo 52. The decisions of the ECtHR, http://cmiskp.echr.coe.int/tkp197/search.asp.

Harris et al., supra nota.

${ }^{36}$ Idem.

${ }^{37}$ Véanse, por ejemplo, los tres casos siguientes decididos por el TEDH: Zdanoka vs. Latvia (16 de marzo 2006), Adamsons vs. Latvia (24 de junio de 2008) y Tanase vs. Moldova (27 de abril de 2010). Para una extensa valoración de la jurisprudencia del TEDH sobre el derecho a la libre elección véase Harris et al., supra nota.

${ }^{38}$ Tal interpretación considera el CEDH (incluidos sus Protocolos) como "un instrumento vivo que... debe ser interpretado a la luz de las condiciones actuales" siempre que una norma haya encontrado amplia y suficiente aceptación entre los Estados miembros como para afectar el sentido del Convenio; TEDH, Tyrer vs. UK, 25 de abril de 1978, serie A, Harris et al., supra nota, p. 7 y ss.
} 
Este obra forma parte del acervo de la Biblioteca Jurídica Virtual del Instituto de Investigaciones Jurídicas de la UNAM www.juridicas.unam.mx

DOl: http://dx_doi.org/10.22201/ijj_24487910e_2015.7.10083

del Consejo de Europa, para escudriñar si un nuevo estándar europeo había surgido. ${ }^{39}$ Por lo que, en dos casos recientes referidos al derecho de voto de dos privados de libertad, Hirst vs. Reino Unido (2005) ${ }^{40}$ y Frodl vs. Austria (2010), ${ }^{41}$ el TEDH recurrió a una interpretación más amplia del artículo 30., fundamentado en una interpretación dinámica del CEDH como un instrumento vivo, a pesar de que la legislación de los Estados partes del CEDH sobre el asunto, no fue uniforme. ${ }^{42}$ En estos dos casos el Tribunal consideró que la privación ex lege del derecho del voto a las personas privadas de libertad de ambos países, constituye una violación del artículo 3o. antes citado. ${ }^{43}$

El desarrollo dinámico de las normas electorales por parte del TEDH refleja una cierta tensión con su mandato de interpretar y aplicar el CEDH, y sus protocolos ${ }^{44}$ con el fin de garantizar el cumplimiento de las obligaciones de los Estados partes: ${ }^{45}$ el TEDH ha desarrollado estándares electorales, que en algunos casos ni siquiera se han fundado en una norma europea común, especialmente en los casos del derecho al voto de los privados de libertad Hirst y Frodl. De hecho, la opinión mayoritaria del TEDH en Hirst fue criticada en una opinión disidente conjunta de eminentes jueces como Wildhaber y Costa, quienes afirman que "el TEDH no es un legislador y debe tener cuidado de no asumir funciones legislativas". ${ }^{46}$ Tal desarrollo dinámico de las normas parece especialmente delicado en el campo de los derechos políticos, los cuales se encuentran intrínsecamente

\footnotetext{
${ }^{39}$ TEDH, Tanase vs. Moldova, supra nota, párrafos 87 y ss.

${ }^{40}$ TEDH, Hirst vs. The United Kingdom, 6 de octubre de 2005; véase la sentencia pionera del TEDH, Greens and M.T. vs. UK where the ECtHR reiterated its findings in Hirst (ECtHR, Greens and M.T. vs. UK, 23 de noviembre de 2010, pp.77 y ss.

${ }^{41}$ ECtHR, Frodl vs. Austria, 8 de abril de 2010.

${ }^{42}$ Joint Dissenting Opinion of the Judges Wildhaber, Costa, Lorenzen, Kovler and Jebens to Hirst vs. UK, párrafo 6.

${ }^{43}$ Del mismo modo en Hirst, el TEDH tuvo como objetivo identificar "Ias normas europeas comunes". Sin embargo, el Tribunal ignoró que solamente 18 de los 45 Estados contratantes no tenían restricciones al derecho al voto de los privados de libertad. (Ver crítica en Voto Disidente Conjunto de Hirst, Identificación, párrafo 6; Hirst vs. Reino Unido, supra nota 39, párrafos 33 y ss.

${ }^{44}$ Artículo 32, CEDH.

${ }^{45}$ Artículo 19, CEDH.

${ }^{46}$ Véase Criticism in the Joint Dissenting Opinion to Hirst, supra nota, párrafos 6 y 9.
} 
Este obra forma parte del acervo de la Biblioteca Jurídica Virtual del Instituto de Investigaciones Jurídicas de la UNAM www.juridicas.unam. $\mathrm{mx}$

DOl: http://dx.doil.org/10.22201/ijj.24487910e.2015.7.10083

conectados a determinados acuerdos electorales de un país y a algunas excepciones constitucionales.

También resulta difícil afianzar el desarrollo y la aplicación de las normas electorales internacionales a la práctica de la observación electoral de la OIDDH, sustentado en el consentimiento estatal y al amplio mandato de la institución.

B. La práctica de la observación electoral de la OSCE-OIDDH

El fundamento principal para la realización de misiones de observación electoral de la OIDDH es que exista una invitación permanente a los Estados participantes conforme a la Conferencia sobre la Seguridad y la Cooperación en Europa, en adelante CSCE (Documento de Copenhague), el cual establece en el párrafo 80. ${ }^{47}$ en términos amplios y generales lo siguiente: "los Estados participantes consideran que la presencia de observadores, ya sean extranjeros o nacionales, puede realzar el proceso electoral de los Estados en que se celebren elecciones. Por consiguiente, permitirán a observadores de otros Estados participantes de la CSCE... dichos observadores no interferirán en los procesos electorales". ${ }^{48}$

De acuerdo a la práctica de la OSCE, no se requiere invitación adicional para las misiones de observación que se desplegarán en una elección específica. Los compromisos asumidos por los Estados de la OSCE relativos a las normas de los procesos electorales nacionales, contenidos en el Documento de Copenhague incluyen: los principios del sufragio universal e igual, el secreto del voto, la necesidad de elecciones libres en intervalos razonables, la disputa por el voto popular de los puestos de al menos una cámara de la legislatura nacional, el establecimiento libre de partidos políticos, la necesidad de garantizar una campaña electoral en un ambiente libre y justo, y el acceso sin impedimentos para los medios de comunicación de todas las agrupaciones políticas. ${ }^{49}$

\footnotetext{
${ }^{47}$ Copenhagen Document, supra nota.

${ }^{48}$ Id., párrafo 8. El compromiso para recibir observadores se fortaleció en las resoluciones posteriores de la cumbre y en los documentos finales, véase infra nota y ss.

${ }^{49}$ Copenhagen Document, supra nota, párrafo 7; 1990, Charter of Paris, Evers, F., “OSCE Election Observation. Commitments, Methodology, Criticism", 15 OSCE Yearbook 2009, pp.235, 236 y ss.
} 
Este obra forma parte del acervo de la Biblioteca Jurídica Virtual del Instituto de Investigaciones Jurídicas de la UNAM www.juridicas.unam.mx

Aunque los documentos de la OSCE no constituyen tratados internacionales en el sentido clásico y formal — de hecho, su naturaleza jurídica es aún controversial $-{ }^{50} \mathrm{se}$ basan en el consenso de todos los Estados participantes de la OSCE, ${ }^{51}$ con lo cual están al menos destinados a la producción de compromisos fuertes y efectivos. ${ }^{52}$

La OIDDH es la institución encargada del monitoreo de las elecciones. Fue "creada por los Estados participantes para ayudarles a cumplir sus compromisos en la dimensión humana, incluido los relacionados con las elecciones". ${ }^{53}$ Sin embargo, el mandato concreto de la OIDDH y las acciones que puede emprender se describen vagamente. La OIDDH tiene el mandato de llevar a cabo "el seguimiento exhaustivo de las elecciones" 54 y desempeñar un "papel preponderante en la supervisión de elecciones, antes, durante y después de las elecciones". ${ }^{55}$ En la Carta sobre la Seguridad Europea, aprobada en la

${ }^{50}$ Algunos académicos consideran los documentos de la OSCE como tratados internacionales, pero sin la responsabilidad clásica y jurídica del Estado, Klabbers, J., The Concept of Treaty in International Law, 1996, p.126; otros ven los documentos como soft law con efectos políticos vinculantes, Fastenrath, U., “The Legal Significance of CSCE-OSCE Documents", OSCE Yearbook, 1995-1996, pp.411 y 418; Schweisfurth, T., "Die juristische Mutation der KSZE", en Beyerlin, U., (ed.), Recht zwischen Umbruch und Bewahrung, 1995, pp. 213 y 224; nuevamente otros los consideran compromisos políticos estrictamente no vinculantes, Ipsen, K. y Epping, Völkerrecht, V., 5ta. ed., 2004, pp.529 y ss; Farahat, A., “Regulating Minority Issues through Standard Setting and Mediation: the Case of the High Commissioner on National Minorities", en Von Bogdandy, A., Wolfrum. R. et al. (eds.), The Exercise of Public Authority by International Institutions. Advancing International Institutional Law, 2009, pp. 343 y 346.

${ }^{51}$ De acuerdo con Röben, la unanimidad favorece la legitimidad, Röben, V., “What about Hobbes? Legitimacy as a Matter of Inclusion in the Functional and Rational Exercise of International Public Power", en Wolfrum, R. y Röben, V. (eds.), Legitimacy in International Law, 2008, pp. 353, 357 y ss.

${ }^{52}$ Farahat, A., para los resultados comparativos sobre el Alto Comisionado para las Minorías Nacionales, supra nota.

${ }^{53}$ EOM Handbook, supra nota 7. OIDDH fue originalmente establecida como la Oficina para Elecciones Libres mediante una decisión adoptada en la Cumbre de la CSCE celebrada en París en 1990, predecesor de la OSCE. ${ }^{54}$ Cuarta Reunión del Consejo de Ministros de la CSCE, Roma, 30 de noviembre 1993, “CSCE and the New Europe-Our Security is Indivisible", http://Www.osce.org/documents/mcs/1993/11/4165_en.pdf, para. IV.4.

${ }^{55}$ Cuarta Cumbre de Jefes de Estado, Budapest, “Documento de Budapest 1994: Towards a Genuine Partnership in a New Era", http://www.osce.org/documents/mcs/1994/12/4048_en.pdf, Ch. VIII, párrafo 12. Véase también aclaraciones aportadas por la Decisión 19/06 del Consejo Ministerial de la OSCE de 2006, Bruselas, 05 de diciembre de 2006, "Strengthening the Effectiveness of the OSCE", sec. 2, párrafo 13. 
Este obra forma parte del acervo de la Biblioteca Jurídica Virtual del Instituto de Investigaciones Jurídicas de la UNAM www.juridicas.unam.mx

DOl: http://dx.doi.org/10.22201/iij.24487910e_2015.7.10083

Cumbre OSCE de Estambul en 1999, los Estados participantes también se comprometieron a seguir puntualmente las recomendaciones de la OIDDH. ${ }^{56}$

Sobre la base de la permanente invitación de Copenhague y los subsecuentes documentos de la OSCE, las misiones de observación electoral de la OIDDH analizan el proceso electoral de un país desde diferentes perspectivas: jurídica, electoral y política, pero además desde la perspectiva de los medios de comunicación. Su evaluación se centra no sólo en la jornada electoral sino que incluyen las fases pre y post-electoral. Las conclusiones de las misiones se publican en declaraciones e informes, ${ }^{57}$ que en considerables detalles concretizan los compromisos del Documento de Copenhague en cuanto a su aplicación a una elección en específico. ${ }^{58}$ Los informes electorales también contienen recomendaciones y directrices de acción para los actores no estatales, como los partidos políticos y los medios de comunicación. ${ }^{59}$ La publicación de la declaración preliminar se realiza inmediatamente después de la jornada electoral, la cual por lo general recibe una fuerte atención de los medios y ejerce una importante presión sobre las autoridades nacionales para que las elecciones se realicen de conformidad con las normas internacionales. ${ }^{60}$

Por consiguiente, las misiones de observación electoral de la OIDDH ejercen autoridad,

56 OSCE Istanbul Summit, "Istanbul Document 1999: Charter for European Security", http://www.osce.org/documents/mcs/1999/11/4050_en.pdf, párrafo 26.

${ }^{57}$ OSCE/ODIHR Website, http://www.osce.org/odihr-elections/14207.htm/.

${ }^{58}$ Evers se refiere a "la implementación práctica de las normas interpretadas" que se desarrollan como consecuencia de la interpretación y aplicación por parte de la OIDDH y los Estados participantes, "creando una especie de derecho consuetudinario en el proceso"; Evers, supra nota pp. 236 y 239). Véase sección 3.2 infra; Binder, C., "International Election Observation by the OSCE and the Human Right to Political Participation", 13 European Public Law, 2007, pp. 133, 148 y ss.

${ }^{59}$ Véanse las Recomendaciones de la OIDDH sobre las actividades de la administración electoral, y su referencia a los programas de educación electoral, sobre la participación de las mujeres y las minorías, en los informes de observación electoral, supra nota. Además de la obligación de divulgar e informar sobre los fondos de las campañas, así como las normas relativas a las campañas en los medios de comunicación de la OSCE / OIDDH, compromisos existentes para las elecciones democráticas en los Estados participantes de la OSCE, Varsovia, 2003, http://www.osce.org/odihr/publications.htm/?/si=true\&limit=10\&grp=243, párrafos 7.8, $7.13,7.14$.

${ }^{60}$ Binder, supra nota, p.150. 
Este obra forma parte del acervo de la Biblioteca Jurídica Virtual del Instituto de Investigaciones Jurídicas de la UNAM www.juridicas.unam.mx

en particular mediante la distribución de información y por medio de su valoración sobre una elección; ${ }^{61}$ provocan estados con bastante eficacia para aplicar las normas electorales, en términos de Richard Chambers y Anne van Aaken "gobernanza a través de la información". ${ }^{62}$ Mientras que los observadores electorales se centran en los aspectos técnicos del proceso y se guían por los principios de imparcialidad y no injerencia, ellos pueden (des)legitimar gobiernos a través de sus observaciones. Este es quizás el mejor ejemplo de las llamadas "revoluciones de color" en Georgia (2003), Ucrania (2004) y Kirguistán (2005), donde las críticas de los observadores de la OIDDH dieron credibilidad a las acusaciones de fraude presentadas por la oposición, con lo cual contribuyeron a un cambio de régimen. ${ }^{63}$

El impacto potencial de apreciables repercusiones que tiene una misión de evaluación, se encuentra en cierto modo en contraste con las bases indeterminadas de las actividades de los observadores; a saber, de la invitación vagamente formulada de los observadores electorales en el Documento 1990 de Copenhague y del amplio mandato de la OIDDH. Esta situación se agrava por el hecho de que, políticamente, es imposible para los Estados participantes de la OSCE retirar la invitación permanente para observadores electorales que figuran en el Documento de Copenhague. De hecho, las actividades de la OIDDH fueron impugnadas dentro de la OSCE. Desde 2003/04 Rusia y otros miembros de la Comunidad de Estados Independientes (CEI) han criticado constantemente a la OIDDH por la aplicación de “dobles estándares” y el ejercicio de una "dudosa autonomía". ${ }^{64}$

${ }^{61}$ Van Aaken, A. y Chambers, R., “Accountability and Independence of International Election Observers”, 2009, 6 International Organizations Law Review, pp. 541-580.

${ }^{62}$ Idem, p. 28.

${ }^{63}$ Meyer-Resende, M., “Exporting Legitimacy: The Record of EU Election Observation in the Context of EU Democracy Support", CEPS Working Document March 2006, 12; Binder, supra nota, p.150.

${ }^{64}$ Cfr. La declaración del ministro de Relaciones Exteriores de Rusia: “...la autonomía de la OIDDH se ha convertido en una ausencia total de control y de los gobiernos decentes no pueden aceptar esto; de otra manera los miembros de la OSCE también querrán buscar <<autonomía>> de la OIDDH", Lavrov, S., Minister for Foreign Affairs of the Russian Federation, at the OSCE Ministerial Council, Ljubljana, 5-6 diciembre de 2005, http://Www.osce.org/conferences/mc_2005.html?page=documents\&group=author. Véase también la Declaración de Moscú sobre el Estado de Asuntos de la OSCE, donde la OIDDH fue acusada de confundirse a través de la politización, Moscow Declaration on the State of Affairs in the OSCE, 8 de julio de 2004, 
Este obra forma parte del acervo de la Biblioteca Jurídica Virtual del Instituto de Investigaciones Jurídicas de la UNAM www.juridicas.unam.mx

\section{Apreciación}

La evaluación mostrada supra nos indica que tanto el TEDH como las misiones de observación electoral de la OIDDH han desarrollado y aplicado las normas electorales internacionales posteriores a 1989 con un dinamismo creciente. De este modo, ambas instituciones han extendido la influencia del derecho internacional y contribuido al hecho de que "el derecho internacional haya penetrado el derecho nacional en materia electoral" europeo.

Las acciones del TEDH y la OIDDH tienen alguna base en el consentimiento estatal. El TEDH ejerce su jurisdicción solamente cuando un Estado ha ratificado el CEDH y su primer Protocolo, y la OSCE participa en el despliegue de observadores electorales cuando los Estados han dado su consentimiento para la adopción de los documentos pertinentes de la OSCE. Sin embargo, el consentimiento original de los Estados no abarca todos los aspectos de las acciones de las instituciones mencionadas. Esto puede explicarse por la indeterminación del marco y la normativa aplicable (es decir, normas electorales vagas, o el amplio mandato de la OIDDH), que proporcionan insuficiente orientación para las actividades de las instituciones. ${ }^{65}$ Además, la generalidad y la manera en que los Estados aceptan la autoridad y jurisdicción permanente del TEDH y la invitación permanente de observadores, reduce el papel legitimador del consentimiento original, especialmente por el activismo de ambas instituciones. ${ }^{66} \mathrm{El}$ vínculo legal entre el consentimiento y el continuo ejercicio de su autoridad se ve debilitado, ya que como se

PC.DEL/630/04, un texto aprobado por todos los Estados de la CEI, excepto Georgia, Azerbaijan y Turkmenistan; Ghébali, V-Y., "Debating Election and Election Monitoring Standards at the OCSE: Between Technical Needs and Politicization", 11 OSCE Yearbook, 2005, p.215.

${ }^{65}$ La determinación de una norma es, según Thomas Franck, vital para medir la precepción de su legitimidad, ya que el grado de su aplicación corresponderá con el consentimiento original concedido; Franck, T., “The Power of Legitimacy and the Legitimacy of Power: International Law in an Age of Power Disequilibrium", 100 American Journal of International Law, 2006, pp. 88 y 94; Franck, T., Fairness in International Law and Institutions, 1995, pp. 30 y ss.

${ }^{66} \mathrm{Al}$ considerar el papel legitimador del consentimiento estatal, Bodansky distingue entre consentimiento específico respecto a las obligaciones o decisiones particulares (por ejemplo, mediante la ratificación de un tratado con obligaciones específicas o la aceptación de la jurisdicción de un tribunal a un caso concreto) y el consentimiento general, referido a un sistema permanente de gobierno, que una vez establecido desarrolla una vida jurídica propia (la presentación general a la jurisdicción de un tribunal; la ratificación de la Carta de la ONU, con sus instituciones permanentes); Bodansky, supra nota, p. 604; Wolfrum, supra nota, p. 2042. 
Este obra forma parte del acervo de la Biblioteca Jurídica Virtual del Instituto de Investigaciones Jurídicas de la UNAM www.juridicas.unam.mx

ha dicho el TEDH ha ampliado considerablemente su criterio en una interpretación extensiva del artículo 3o. del primer Protocolo (P) del CEDH e igualmente, las múltiples actividades de los observadores electorales de la OIDDH se basan en un amplio e impreciso mandato concedido a la OIDDH y en la invitación general de los observadores de los Estados.

El extenso ejercicio de actividades de ambas instituciones, es particularmente sensible visto el potencial impacto que tienen los resultados en el nivel nacional. De hecho, los juicios del TEDH pueden afectar el marco legal electoral de los Estados en cuestiones como el derecho al voto de los nacionales residenciados fuera del país, ${ }^{67}$ el umbral de votos necesarios para obtener escaños en el parlamento ${ }^{68} \mathrm{o}$ el derecho de los ex comunistas a presentarse en las elecciones en aquellos países que han emergido de un pasado comunista. ${ }^{69}$ Como se mencionó, las misiones de observación electoral de la OIDDH pueden des-legitimar gobiernos a través de sus evaluaciones. Por lo tanto, el activismo de ambas instituciones en materia electoral ha creado una situación de alguna manera paradójica, al permitir - a través de la aplicación de las normas electorales- la realización del derecho emergente a la democracia. Esto sin embargo, a riesgo de valorarse como posibles intervenciones graves en los ordenamientos jurídicos nacionales.

Un análisis sobre la presunta insuficiencia de legitimidad de origen, así como del considerable impacto potencial del dinamismo de las instituciones en materia electoral, con una mirada más amplia sobre la legitimidad de sus actividades será realizado en la siguiente sección. Más allá del consentimiento estatal, ¿de qué otra manera puede justificarse?

\section{PERSPECTIVAS DE LEGITIMIDAD DE LAS NORMAS ELECTORALES INTERNACIONALES: UNA COMPARACIÓN}

Recordando la definición de legitimidad, como "la cualidad que lleva a las personas (o

${ }^{67}$ Sitaropoulos y otros, vs. Greece, 8 de julio de 2010.

${ }^{68}$ En Yumak and Sadak vs. Turkey (8 de julio de 2008), el TEDH tuvo que plantearse el desafío del límite del $10 \%$ de los votos requeridos para ganar un escaño en el parlamento turco, aunque el Tribunal no estableció una violación en este caso. Véase Golubok sobre la importancia de que el TEDH proceda a examinar los sistemas electorales; Golubok, supra nota, pp. 376 y ss.

${ }^{69}$ Zdanoka vs. Latvia, supra nota, Adamsons vs. Latvia, supra nota. 
Este obra forma parte del acervo de la Biblioteca Jurídica Virtual del Instituto de Investigaciones Jurídicas de la UNAM www.juridicas.unam.mx

Estados) a aceptar autoridad, independientemente de la coerción, el interés propio, o la persuasión racional, a causa de una sensación general de que se justifica", en el entendido de que no es sólo una justificación general, y el requisito para el ejercicio de la autoridad, ${ }^{70}$ la fuerza de una norma es percibida también como legítima por el hecho de que conduce, en términos de Thomas Franck, a aquellos a los que se dirige hacia el cumplimiento consensual. ${ }^{71}$ Esto parece especialmente importante en el derecho internacional, para lo cual a menudo no hay mecanismos eficaces de cumplimiento.

Frente a la creciente insuficiencia de consentimiento del Estado para justificar la autoridad de las instituciones internacionales, el interés doctrinal en la legitimidad ha crecido. ${ }^{72}$ Los académicos han sugerido diferentes modelos y elementos que pueden provocar legitimidad al ejercicio de una autoridad particular. ${ }^{73}$ Ellos distinguen ampliamente entre la fuente, el procedimiento y los enfoques orientados a los resultados, o una combinación de los mismos. ${ }^{74}$ Dicho de otra manera, la autoridad puede ser legitimada por su origen o fuente (tradicionalmente el consentimiento del Estado), también puede ser considerada legítima una acción, cuyos procedimientos sean adecuados y justos, o debido a su éxito en la producción de los resultados esperados. ${ }^{75}$ Aspectos funcionales, incluyendo la experiencia de la respectiva institución o la existencia de mecanismos de rendición de cuentas adecuadas, también pueden ser tomados en consideración. ${ }^{76}$

\footnotetext{
${ }^{70}$ Bodansky ubica la legitimidad "en algún lugar entre la persuasión y la compulsión racional" como base para la acción, Bodansky, supra nota, p.707.

${ }^{71}$ Franck, T., The Power of Legitimacy among Nations, 1990.

${ }^{72}$ Además de los ya mencionados, Bodansky, supra nota; Koskenniemi, M., “ Legitimacy, Rights and Ideology: Notes Towards a Critique of the New Moral Internationalism", 7 Associations, 2003, p.349.

${ }^{73}$ Wolfrum, supra nota, p. 2040; Bodansky, supra nota, pp. 611 y ss. Como se ha indicado, está más allá del alcance de este documento para hacer referencia a otros enfoques de legitimación como conceptos de democracia transnacional.

${ }^{74}$ Wolfrum, supra nota, 2040; Bodansky, supra nota, p. 612.

${ }^{75}$ Id; Delbrück, supra nota, p.42.

${ }^{76}$ Bodansky distingue entre legitimidad legal, la legitimidad participativa, y la legitimidad de expertos, Id., p. 623. Treves establece categorías específicas para evaluar la legitimidad de las decisiones judiciales, incluyendo la forma en que se estableció el órgano judicial; en relación con los miembros de los órganos
} 
Este obra forma parte del acervo de la Biblioteca Jurídica Virtual del Instituto de Investigaciones Jurídicas de la UNAM www.juridicas.unam.mx

En la siguiente parte se abordará el procedimiento (3.1) y la obtención de resultados (3.2) relacionados con las dimensiones de la legitimidad, con el fin de evaluar y comparar el desarrollo, y aplicación de las normas electorales internacionales, tanto en la legislación sobre derechos humanos como en la práctica de la observación electoral. ${ }^{77}$ Debido a las limitaciones de espacio sólo se destacarán los aspectos más relevantes, conscientes de que la legitimidad no es una cuestión de todo o nada, sino de más o de menos.

\section{Legitimidad procesal}

Como se ha dicho, la autoridad puede ser legitimada porque tiene implícita procedimientos que se consideran adecuados y justos, ${ }^{78}$ incluida la composición de la autoridad que decide y los procedimientos que guían la deliberación (toma de decisiones).

Para empezar, la composición de ambas instituciones establece una base razonable para un desarrollo y aplicación imparcial e independiente de las normas electorales. El TEDH se beneficia sobre todo a partir de su carácter judicial, con jueces que gozan de garantías judiciales de imparcialidad, independencia y conocimientos jurídicos. ${ }^{79} \mathrm{La}$ participación de la Asamblea Parlamentaria del Consejo de Europa en la elección de los magistrados aporta cierta legitimidad democrática. ${ }^{80}$ Los requisitos para la

judiciales; la base de la jurisdicción; la forma en cómo se alcanzan los juicios; las características de la decisión y los efectos de la decisión, Treves, supra nota, pp.171 y ss.

77 Del mismo modo, escapa del alcance de este documento abordar la legitimidad de las normas subyacentes-las normas electorales aplicables, aunque esto parece menos problemático, ya que los Estados lo han convenido en general a través de su ratificación del CEDH y su primer $\mathrm{P}$, así como en la adopción del Documento de Copenhague. Véanse, Fuller, L., The Morality of Law, Segundo rev ed, 1969; Brunnée, J. y Toope, S., que, basándose en la teoría de Lon Fuller, propone la moral interna fundada en criterios tales como la esquivación de la contradicción, la generalidad y la congruencia con las normas subyacentes, Brunnée, J. y Toope, S., "International Law and Constructivism: Elements of an Interactional Theory of International Law" 39 Columbian Journal of Transnational Law, 2000, p. 19.

${ }^{78}$ Wolfrum, supra nota, p. 2040.

${ }^{79}$ Artículos 21 y 23.1 CEDH. La independencia de los jueces se fortaleció aún más con la entrada en vigor del Protocolo Núm. 14 en junio de 2010, que amplió el mandato de 9 años y abolió la posibilidad de reelección.

80 De conformidad con el artículo 22 del CEDH, la Asamblea Parlamentaria puede elegir sobre la base de una lista de tres candidatos propuestos por un Estado. Von Bogdandy, A. y I Venzke, "In Whose Name? An 
Este obra forma parte del acervo de la Biblioteca Jurídica Virtual del Instituto de Investigaciones Jurídicas de la UNAM www.juridicas.unam.mx

independencia y la imparcialidad de los observadores de la OIDDH están menos formalizados. ${ }^{81}$ Sin embargo, un código de conducta ${ }^{82}$ que debe ser firmado por todos los observadores, les obliga a la imparcialidad y un alto profesionalismo. ${ }^{83}$ Por otra parte, los miembros del equipo central son expertos independientes con un alto grado de conocimientos técnicos en materia electoral, por lo que generalmente se considera una salvaguardia contra la politización. ${ }^{84} \mathrm{El}$ equipo central es reclutado en una competencia abierta entre una variedad de Estados. ${ }^{85}$ Por último, la composición multinacional de las misiones de observación electoral de la OIDDH proceden de todos los Estados participantes, con un máximo del 10\% de observadores de un país, y nadie puede observar las elecciones en su propio país, ${ }^{86}$ favoreciéndose una observación equilibrada e imparcial.

En cuanto a los procedimientos involucrados para llegar a un juicio, se puede distinguir aproximadamente entre las garantías procesales destinadas para llegar a una

Investigation of International Courts' Public Authority and its Democratic Justification', 2010, pp. 37 y ss., http://ssrn.com/abstract $=1593543$

${ }^{81}$ Misk, J., "Standardizing the Principles of International Election Observation", 13 Vanderbilt Journal of Transnational Law, 2010, p. 763.

82 Declaration of Principles for International Election Observation and Code of Conduct for International Election Observers, 27 de octubre 2005, http://ec.europa.eu/europeaid/observer/declaration_of_principles_code_of_conduct_en.pdf.

${ }^{83}$ Venice Commission, "Guidelines on the Internationally Recognized Status for Election Observers", CDL-AD (2009)059, Strasbourg, 14 de diciembre de 2009, http://Www.venice.coe.int/docs/2009/CDL-AD (2009)059e.asp.

${ }^{84}$ A favor de la legitimidad por expertos independientes Wolfrum, tomando como el ejemplo de la Comisión Jurídica y Técnica de la Autoridad Internacional de los Fondos Marinos, Wolfrum, supra nota, p. 2045. Para críticas véase, Koskenniemi, M., "Global Governance and Public International Law”, 37 Kritische Justiz, 2004, p.241; Klabbers, J., “Two Concepts of International Organization”, 2 International Organizations Law Review, 2005, p. 277; Bodansky, supra nota, pp.718 y ss.

${ }^{85}$ Algunos ven aún margen para mejorar la transparencia de los procedimientos de contratación. Véanse, The OSCE Ministerial Council Decision 2006, supra nota; ODIHR's reply: OSCE/ODIHR, “Common Responsibility. Commitments and Implementation", noviembre de 2006, p. 51, http://www.osce.org/publications/odihr/2006/11/22321_761_en.pdf.

${ }^{86}$ Sobre la estructura de las misiones de observación electoral véase, Election Observation Handbook, supra nota, pp. 37 y ss. 
Este obra forma parte del acervo de la Biblioteca Jurídica Virtual del Instituto de Investigaciones Jurídicas de la UNAM www.juridicas.unam. $\mathrm{mx}$

DOl: http://dx.doil.org/10.22201/iij.24487910e_2015.7.10083

decisión que sea normativamente correcta y las capacidades de las instituciones para llegar a decisiones integrales. En cuanto a lo primero, el TEDH encuentra legitimidad en sus requisitos generales de justicia procesal. ${ }^{87}$ La técnica interpretativa del TEDH, fundamentada en la interpretación dinámica del artículo 3o. del primer Protocolo (P) del CEDH en la detección de una norma europea común ${ }^{88}$ parece, si se aplica correctamente y soportado en cierto consenso europeo (que sin duda no era el caso en Hirst vs. Reino Unido), ${ }^{89}$ una concretización adecuada de una disposición indeterminada del Convenio sobre los derechos políticos: la metodología coherente favorece la consistencia. Del mismo modo, la posible remisión a la Gran Sala ${ }^{90}$ en segunda instancia, de casos que plantean cuestiones jurídicas graves sobre intereses generales, contribuye a la legitimidad, en virtud de su composición, integrada por 17 jueces en funciones. Varios casos relativos a los derechos políticos corresponden a decisiones de la Gran Sala. Aún así, la legitimidad de tales juicios se reduce posiblemente a asuntos relacionados con opiniones disidentes fuertes, como fue el caso de Hirst vs. Reino Unido.

Mientras que el TEDH tiene fuertes garantías procesales y técnicas metodológicas para llegar a decisiones normativamente correctas y esto en consecuencia se traduce en el desarrollo de normas electorales legitimadas, sus posibilidades de investigación son limitadas. ${ }^{91}$ El Tribunal valora los hechos según lo han establecido los tribunales nacionales sobre la base de las solicitudes presentadas por escrito por las partes, pero además porque carece de recursos..$^{92}$ Sólo de manera excepcional se involucra en los

\footnotetext{
${ }^{87}$ Tales como las audiencias públicas, artículo 40, CEDH; véase también la posibilidad de permitir la intervención de terceros, de conformidad con el artículo 36, CEDH.

${ }^{88}$ Tanase vs. Moldova, supra nota, pp. 87 y ss; Hirst vs. UK, supra nota, pp.33 y ss.; the Court's comparative law approach also in Yumak and Sadak vs. Turkey, supra nota, pp. 61 y ss. Véase generally supra, section 2.2.1.

${ }^{89}$ Supra, section 2.2.1.

${ }^{90}$ Artículo 43, CEDH.

${ }^{91}$ ECtHR Hirst vs. UK, supra nota; ECtHR, Zdanoka vs. Latvia, supra nota. Yumak and Sadak vs. Turkey, supra nota.

${ }^{92} \mathrm{Si}$ bien las investigaciones de oficio son, en principio, posibles de conformidad con el artículo 38 CEDH, que rara vez se realizan.
} 
Este obra forma parte del acervo de la Biblioteca Jurídica Virtual del Instituto de Investigaciones Jurídicas de la UNAM www.juridicas.unam.mx

DOl: http://dx.doi.org/10.22201/iij.24487910e.2015.7.10083

hechos o mediante visitas in situ..$^{93}$ De hecho, esto va más allá de las capacidades de una institución judicial internacional para obtener una imagen completa de una elección interna, sin perjuicio de la muy valorada práctica liberal del TEDH, sobre la admisibilidad de amicus curiae, que aportan nuevos datos sobre la situación de un país. ${ }^{94}$ Esto puede obstaculizar la capacidad del TEDH en el sentido de recurrir a un examen exhaustivo de los hechos, especialmente si se tiene en cuenta la complejidad de los procesos electorales y la variedad de actores involucrados. La ponderación de intereses individuales con los relevantes intereses del Estado, puede prevenir consecuencias, como se ha demostrado por ejemplo, en la discutida decisión del TEDH en el caso Sukhovetskyy vs. Ucrania. ${ }^{95}$ En la sentencia, el TEDH no encontró una violación de los derechos políticos de la demandante, a pesar de la elevada y desproporcionada fianza exigida, que sin duda le habría impedido presentarse a las elecciones. ${ }^{96}$

La garantía procesal que asegura la adecuada normativa y la ausencia de sesgo en las evaluaciones de las misiones de observación electoral de la OIDDH, es la metodología elaborada y formalizada de la institución. ${ }^{97}$ Se establece en "manuales de observación" que proporcionan las directrices detalladas para las misiones de observación electoral, con el fin de que realicen su trabajo de una manera que respete los principios de imparcialidad y no injerencia en el proceso electoral. En respuesta a las críticas, la OIDDH también ha diversificado la gama de países a los que las misiones de observación se despliegan, los cuales incluye no sólo aquellos que emergen de un pasado no democrático sino también - aunque a una escala menor - a aquellos donde rigen democracias más consolidadas. ${ }^{98}$

Aunque los informes de las misiones se preparan en colaboración con la sede

${ }^{93}$ Harris et al; supra nota, pp.846 y ss., para mayor referencias.

${ }^{94}$ Artículo 44 del Reglamento del TEDH. (Véase, el artículo 854 para mayores referencias.) Por ejemplo, en Hirst vs. UK se observa la positiva presentación de escritos amicus curiae como medio para aumentar la legitimidad subjetiva de los hallazgos; Hirst vs. UK, supra nota, pp. 53 y ss.

${ }^{95}$ ECtHR, Sukhovetskyy vs. Ukraine, 2006-VI, 44 EHRR 1185.

${ }^{96}$ Para críticas sobre la decisión, Harris et al; supra nota, p. 721.

${ }^{97}$ EOM handbook, supra nota.Declaration of Principles for International Election Observation, supra nota; Evers, supra nota, pp. 243 y ss.; Aaken-Chambers, supra nota, p. 551.

${ }^{98}$ Evers, supra nota, pp.241 y ss. 
Este obra forma parte del acervo de la Biblioteca Jurídica Virtual del Instituto de Investigaciones Jurídicas de la UNAM www.juridicas.unam. $\mathrm{mx}$

principal de la OIDDH, la independencia de las misiones de observación electoral de su organización "madre" de la OIDDH, reside principalmente en el hecho de que ésta es una institución independiente dentro de la OSCE. ${ }^{99}$ Sin embargo, los mecanismos de rendición de cuentas de las misiones de observación electoral todavía no están suficientemente formalizados. ${ }^{100} \mathrm{El}$ activo más importante de la elección de las misiones de observación es su amplia capacidad para la investigación de los hechos, lo cual se deriva de la presencia de los observadores en todo el país. Las misiones de observación electoral, por lo tanto, fundamentan sus conclusiones en observaciones completas que a su vez se sustentan en contactos con todas las partes interesadas en el proceso electoral (miembros de la comisión electoral, los partidos políticos, representantes de la sociedad civil, etcétera).

\section{Legitimidad sustantiva o del resultado}

La legitimidad sustantiva basada en el resultado - hablando francamente- está asociada al desempeño de la institución que realiza "un buen trabajo en el gobierno", vinculado también con la eficacia. ${ }^{101}$ Sobre la base de la discusión planteada por Treves, referida a la legitimidad de las decisiones judiciales, vamos a abordar en particular las cualidades inherentes, es decir, las características de una decisión, entre ellas la aplicación coherente de la legislación pertinente, la calidad del razonamiento jurídico ${ }^{102}$ y la relación entre una decisión, y su implementación (en términos más generales, en qué medida el resultado es aceptado por la comunidad respectiva). ${ }^{103}$ Un examen de estas dimensiones se desarrollará seguidamente.

Al aplicar el artículo 3o. del primer Protocolo (P) del CEDH, el TEDH invoca su

${ }^{99}$ Id., p.18. Evers amplía en detalles, especificando que la independencia de la OIDDH se basa en documentos de trabajo de la OSCE-OIDDH para llevar a cabo la observación electoral independiente, así como la constante afirmación de la OIDDH de su propia independencia que también fue aceptada por la mayoría de los Estados participantes, mientras que de acuerdo con las Reglas de la OSCE de Procedimiento, la OIDDH no es un órgano de toma de decisiones, sino una estructura ejecutiva o institución de la OSCE, Id., pp.244 y ss.

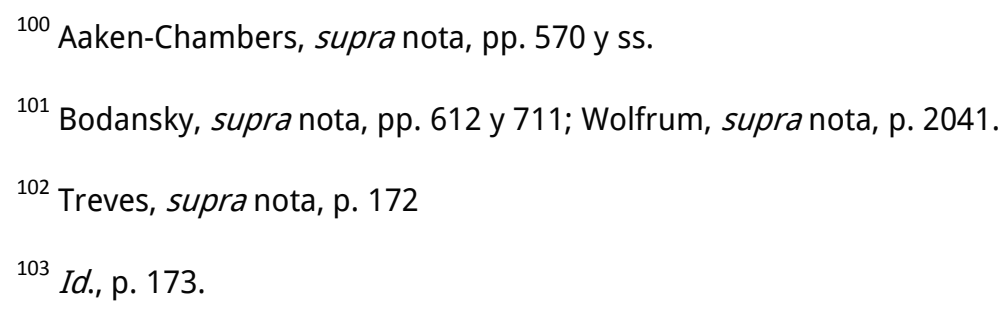


Este obra forma parte del acervo de la Biblioteca Jurídica Virtual del Instituto de Investigaciones Jurídicas de la UNAM www.juridicas.unam.mx

DOl: http://dx.doil.org/10.22201/iij_24487910e.2015.7.10083

jurisprudencia anterior. ${ }^{104}$ Tales referencias son bienvenidas, ya que tienen el efecto práctico de la construcción del sistema con los precedentes, ${ }^{105}$ la promoción de la coherencia de las sentencias del TEDH y la estabilización de las expectativas normativas. ${ }^{106}$ La técnica interpretativa del TEDH de comparar los diferentes ordenamientos jurídicos de los Estados para investigar si una nueva norma europea en cuestión ha surgido - documentado en las consideraciones del TEDH - promueve la transparencia. ${ }^{107}$ Por el contrario, la coherencia de la elaboración de las normas de las misiones de observación de la OIDDH, se ha visto facilitada por las numerosas misiones desplegadas durante los últimos 20 años. Las misiones han desarrollado sistemáticamente las normas electorales indeterminadas en su interpretación y aplicación. El involucramiento de los Estados en la ejecución, ha contribuido al desarrollo de mejores prácticas 0 , en términos Evers, "compromisos interpretativos". ${ }^{108}$ Este derecho no vinculante ${ }^{109}$ establece detallados criterios para evaluar un proceso electoral. Adicionalmente, como las misiones se envían generalmente de manera consecutiva al mismo país, los puntos específicos de referencia se establecen para comparar el desempeño electoral de ese país con respecto a las elecciones anteriores. ${ }^{110}$ Las

${ }^{104}$ Tanase vs. Moldova, supra nota, pp. 104 y ss.; Frodl vs. Austria, supra nota, pp. 22 y ss. Véase la obligación del Tribunal de motivar sus sentencias, artículo 45, CEDH.

${ }^{105}$ El Tribunal no está vinculado formalmente por sus decisiones anteriores. Jacob, M., “Lawmaking Through International Adjudication", 12 German Law Journal, 201, p. 1005, http://Www.germanlawjournal.com/index.php?pageID=11\&artID=1351.

${ }^{106}$ Incluso si una norma es indeterminada, la coherencia general en su aplicación puede, sin embargo, añadir algo a su legitimidad percibida. Según lo declarado por Franck, una regla que es vaga, puede todavía ser percibida como legítima si su aplicación en casos dados, está abierta a un proceso que da especificidad, Franck, supra nota, p. 94.

${ }^{107}$ Ver también el aumento de la referencia del TEDH a las normas de derecho no vinculante, como se muestra por ejemplo en, Sitaropoulos vs. Greece, supra nota, p.44.

${ }^{108}$ Evers se refiere incluso a "una especie de derecho consuetudinario", Evers, supra nota, pp. 236 y 255.

${ }^{109}$ Compromisos existentes supra nota. El documento fue elaborado por un grupo de expertos en derecho internacional con experiencia en prácticas de derecho electoral y derechos humanos en Occidente, así como la CEI y los Estados de la antigua Yugoslavia, y también fue aprobado por el Consejo Permanente. (PC/Dec 509, 5 de diciembre de 2002.)

${ }^{110}$ Informe de la misión de evaluación electoral de la OIDDH Uzbekistan 2009, en el cual los comentarios 
Este obra forma parte del acervo de la Biblioteca Jurídica Virtual del Instituto de Investigaciones Jurídicas de la UNAM www.juridicas.unam.mx

elecciones son así evaluadas de una manera detallada y coherente que mejora la calidad de las conclusiones de una misión de observación electoral.

La evaluación de la relación entre una decisión y su implementación se encuentra en la salvedad obvia de que el cumplimiento por parte de las autoridades estatales de una sentencia sólo puede ser, en el caso de los derechos humanos, una guía parcial respecto de la legitimidad sustantiva de las instituciones, donde en última instancia, los individuos son los destinatarios-beneficiarios. ${ }^{111}$ Esto parece ser aún más claro con respecto a los derechos electorales-políticos, donde la implementación debe servir de autogobierno democrático incluyendo la posible sustitución de los regímenes autoritarios (que podrían oponerse a la ejecución de una decisión). Por lo tanto, la "comunidad de que se trate" alude más evidentemente al caso de los derechos políticos-particulares. En consecuencia, el enfoque especial se pondrá en la eficacia de las instituciones, lo cual significa un mayor cumplimiento.

Sin embargo, cuando se trata de la relación entre una decisión y su implementación, los medios con los que cuenta el TEDH son limitados. En efecto, un Estado está obligado a aplicar las sentencias del Tribunal de conformidad con el artículo 46 del CEDH. Sin embargo, la aplicación efectiva de las normas electorales con respecto a una elección se ve impedida, en primer lugar, por el carácter ex-post de las sentencias del TEDH y en segundo lugar, por el largo tiempo que transcurre entre la presentación de una solicitud y la decisión del TEDH. ${ }^{112}$ Para ejemplificar tenemos el caso Yumak y Sadak vs. Turquía, sobre las elecciones parlamentarias de Turquía de 2002, el cual fue decidido después de las elecciones de $2007 .{ }^{113}$ Por lo tanto, las sentencias del Tribunal parecen más útiles

sobre el desempeño electoral del país, se comparan con anteriores recomendaciones presentadas en el informe sobre las elecciones parlamentarias de 2004 y la evaluación de 2005 sobre el marco legal; OSCE/ODIHR Election Assessment, Final Report, Republic of Uzbekistan, Parliamentary Elections 27 de diciembre de 2009, 7 de abril de 2010, http://Www.osce.org/documents/odihr-el/2010/04/43367_en.pdf; Binder, C.,"Möglichkeiten und Grenzen der Wahlbeobachtung in unsicheren Demokratien am Beispiel Usbekistans", Verfassung und Recht in Übersee, 2010, p. 418.

${ }^{111}$ Bodansky, supra nota Error: No se encuentra la fuente de referencia, en referencia a la creciente importancia de los destinatarios no estatales.

${ }^{112}$ Treves, supra nota, p. 173.

${ }^{113}$ Wolfrum, supra nota, p. 2041: otro aspecto de la legitimidad sustantiva puede ser la eficiencia; si bien como sostiene Wolfrum, esto no debería ser sobrevalorado. 
Este obra forma parte del acervo de la Biblioteca Jurídica Virtual del Instituto de Investigaciones Jurídicas de la UNAM www.juridicas.unam. $\mathrm{mx}$

cuando se trata de abordar insuficiencias específicas en el largo plazo. Pero además, las herramientas del TEDH para promover la aplicación de las normas electorales se ven limitadas. El Comité de Ministros supervisa la ejecución de las sentencias del TEDH y publica los registros de cumplimiento de los Estados en el sitio web del Consejo de Europa. Con la entrada en vigor del Protocolo Núm. 14, las facultades de supervisión del Comité de Ministros se han incrementado. Ahora el Comité puede remitir los casos de incumplimiento al TEDH, ${ }^{114}$ con lo cual se ejerce una presión considerable sobre los Estados para cumplir las decisiones judiciales. No obstante, el TEDH como institución judicial, no tiene los medios para apoyar o asistir técnicamente a un Estado para corregir las deficiencias cuestionadas en su marco electoral. ${ }^{115}$ En la práctica, el cumplimiento de Ios Estados con las sentencias del TEDH parece mixta. ${ }^{116}$ La mayoría de los Estados acataron las sentencias del TEDH o al menos manifestaron su voluntad de hacerlo pero en algunos asuntos se han determinado demoras en la ejecución, ${ }^{117}$ o falta de información al Comité de Ministros sobre el estado de cumplimiento de las decisiones del tribunal. ${ }^{118}$

\footnotetext{
${ }^{114}$ Artículo 46.4, CEDH.
}

${ }^{115}$ De hecho, el Consejo de la Comisión de Venecia de Europa da opiniones legales - a veces junto con la OIDDH - sobre el marco legal electoral de un país y adopta recomendaciones. Véase, la Comisión de Venecia y la OSCE-OIDDH Dictamen conjunto sobre el Proyecto de Ley de enmiendas y suplementos de la Ley de Elección de Consejeros y de los miembros del Parlamento de Montenegro en su forma enmendada, julio de 2006, y 3 y 4 de junio de 2010, http://Www.venice.coe.int/docs/2010/CDL-AD(2010)023-e.asp.

${ }^{116}$ Véase en general el estado de cumplimiento y la ejecución de sentencias, Consejo de Europa, Supervisión de Ejecución. Ejecución de las Sentencias del Tribunal Europeo de Derechos Humanos, http://www.coe.int/t/dghl/monitoring/execution/.

${ }^{117}$ Por ejemplo, a partir de agosto de 2011, el Reino Unido no había aplicado los cambios legislativos necesarios relativos a los derechos de voto de los presos en el seguimiento de Hirst de 2004. (Supervisión de la Ejecución. Ejecución de Sentencias del Tribunal Europeo de Derechos Humanos, 74025/01 Hirst Núm. 2, juicio del 6 de octubre de 2005, Grand Chamber. Interim Resolution CM/ResDH, 2009, p.160; Greens and M.T. vs. UK, supra nota.

${ }^{118}$ A partir de agosto de 2011 el Comité de Ministros sigue a la espera de la información por parte de Letonia de cómo ese país pretende implementar el Juicio Adamsons de 2008 en cuanto a los cambios legislativos necesarios y la posibilidad de que la demandante pueda presentarse a las elecciones. (Supervisión de la Ejecución. Ejecución de Sentencias del Tribunal Europeo, http://www.coe.int/t/dghl/monitoring/execution/Reports/pendingCases_en.asp?CaseTitleOrNumber=adamso 
Este obra forma parte del acervo de la Biblioteca Jurídica Virtual del Instituto de Investigaciones Jurídicas de la UNAM www.juridicas.unam.mx

Las evaluaciones de los procesos electorales realizadas por la OIDDH (por ejemplo, la declaración preliminar, informe final) no son jurídicamente vinculantes, aunque los Estados participantes se hayan comprometido políticamente a dar seguimiento a las recomendaciones de la OIDDH en los documentos pertinentes de la OSCE. ${ }^{119}$ Como se dijo, las misiones electorales ejercen una considerable presión política sobre los Estados para celebrar elecciones de conformidad con las normas internacionales mediante la publicación de sus evaluaciones. El seguimiento en el Consejo Permanente de la OSCE sobre la aplicación de las conclusiones de una misión fue criticado por débil e insuficiente, ${ }^{120}$ por lo que la eficacia de la OIDDH depende de la voluntad política de los Estados y gobiernos. ${ }^{121}$ De hecho, la aplicación de las normas electorales es más efectiva en los Estados que están dispuestos a mejorar su registro electoral, como lo prevén las recomendaciones de los informes de los observadores en el aparte sobre las deficiencias detectadas. Además, la OIDDH en algunos casos, también asiste técnicamente a los Estados en el seguimiento de una misión, ${ }^{122}$ creándose un potencial positivo de implementación cooperativa en el intercambio y el diálogo con el país en cuestión, que debe reforzarse, ${ }^{123}$ mediante la introducción sistemática de un diálogo posterior a las elecciones con los Estados al concluir una misión de observación. ${ }^{124}$ Aún así, la motivación del país sigue siendo vital para que se adopten las recomendaciones de la

ns\&StateCode $=\&$ Section Code $=$

${ }^{119}$ Istanbul Summit, supra nota.

${ }^{120}$ ODIHR Common Responsibility, supra nota, p. 147; Binder, supra nota, p. 157.

${ }^{121}$ E.g. H. Balian, “Ten Years of International Election Assistance and Observation”, 12 Helsinki Monitor, 2001, pp. 197 y 201.

${ }^{122}$ ODIHR Common Responsibility, supra nota, pp. 148 y149. Véase además, varios informes de observación electoral que hacen referencia directa a la posibilidad de la asistencia electoral, por ejemplo, la OSCE/ODIHR, Final Report, Russian Federation, Presidential Election, 14 de marzo de 2004, http://www.osce.org/documents/odihr/2004/06/3033_en.pdf, p.2; para más información sobre la asistencia electoral véase, ODIHR's website, http://Www.osce.org/odihr/?page=elections\&div=assistance; Binder, supra nota, pp. 151 y ss.

${ }^{123} 2003$ Maastricht Ministerial Council, tasking ODIHR para "considerar formas de mejorar la eficacia de su asistencia a los Estados participantes en el seguimiento de [sus] propias recomendaciones," OSCE, $11^{\text {th }}$ Meeting of the Ministerial Council, Maastricht, 1 y 2 de diciembre de 2003, Decisión núm. 5/03, p. 81.

${ }^{124}$ ODIHR Common Responsibility, supra nota, pp. 145 y ss., sobre seguimiento y diálogo post electoral. 
Este obra forma parte del acervo de la Biblioteca Jurídica Virtual del Instituto de Investigaciones Jurídicas de la UNAM www.juridicas.unam.mx

OIDDH. En la práctica, no sólo situación política de un país parece decisiva, sino también la geopolítica. Un estudio de 2001 sobre las tendencias electorales en los países en que la OIDDH observó elecciones, dio como resultado un mayor progreso en la mejora de las normas electorales en Europa Central y Oriental, mejoras lentas pero constantes en los Balcanes, un progreso limitado en el Cáucaso y una situación más difícil en Asia Central. ${ }^{125}$

\section{Apreciación}

Esta valoración indica que en los casos de desarrollo e implementación de normas electorales de ambas instituciones, los aspectos de procedimiento y de resultados relacionados con la legitimidad complementan de alguna manera, la deficiente legitimidad derivada del consentimiento de los Estados. Las actividades de la OIDDH y del TEDH se rigen por garantías procesales destinadas a decisiones imparciales y equilibradas. Del mismo modo, la calidad y la coherencia de los razonamientosevaluaciones legitiman, en principio, el ejercicio de su autoridad. ${ }^{126}$ Estas dimensiones sustantivas y de procedimiento de legitimidad son importantes para guiar al TEDH en el desarrollo de las normas electorales indeterminadas contenidas en el artículo 3o. del primer Protocolo (P) del CEDH, así como también las actividades de la OIDDH, dentro de su amplio mandato. Esto es particularmente crucial para la acción internacional en áreas tan sensibles como los procesos electorales nacionales. Para la OIDDH, ${ }^{127}$ el énfasis continuo y el fortalecimiento de estas dimensiones adicionales de legitimidad parecen de

\footnotetext{
${ }^{125}$ Balian, supra nota, pp. 202 y ss. Para hallazgos similares ver el análisis de las tendencias regionales de Freedom House 2010. Freedom House-A.Puddington, "Freedom in the World 2010: Erosion of Freedom Intensifies", Survey, http://Www.freedomhouse.org/uploads/fiw10/FIW_2010_Overview_Essay.pdf, pp. 7 y ss. Para Europa sudoriental en referencia a la construcción de la democracia, Richter, S., "How Effective Is the OSCE's Promotion of Democracy? Analytical Considerations of the Effectiveness of the Long-Term Missions in South-Eastern Europe", OSCE Yearbook, 2008, p. 191.

${ }^{126}$ Cuando el razonamiento jurídico es pobre, las opiniones disidentes son una herramienta fuerte para resaltar tales deficiencias; Voto Disidente Conjunto; véase por ejemplo, el dictamen disidente en Hirst, supra nota.

${ }^{127}$ Ver responsabilidad común, documento sustancial producido por ODIHR, supra nota. Para la crítica general sobre organizaciones internacionales "agendas ocultas",Ottaway, M., "Should Elections Be the Criterion of Democratization in Africa?", 145 CSIS Africa Notes, 1993, pp. 1 y 3; Abrahamsen, R., Disciplining Democracy: Development Discourse and Good Governance in Africa, 2000.
} 
Este obra forma parte del acervo de la Biblioteca Jurídica Virtual del Instituto de Investigaciones Jurídicas de la UNAM www.juridicas.unam.mx

DOl: http://dx.doi.org/10.22201/iij.24487910e.2015.7.10083

vital importancia, también para responder a Rusia y a las críticas y acusaciones de otros Estados de la CEI, respecto a su "autonomía sin control,"128 que desde otra perspectiva, también se puede ver como intentos de deslegitimar la institución, particularmente después de que la OIDDH había producido sus informes críticos sobre la calidad de los procesos electorales de esos países. ${ }^{129}$

Sin embargo, una comparación en cuanto a la elaboración y aplicación de las normas electorales desde las perspectivas de legitimidad y de procedimiento, relacionadas con la obtención de resultados, pone de manifiesto las divergencias en las fortalezas de las instituciones. El carácter judicial del TEDH y su técnica interpretativa para la construcción de un sistema con precedentes informales y el establecimiento de normas mediante la comparación de los ordenamientos jurídicos de los Estados partes, parece particularmente legítimo del TEDH para la identificación de la evolución de las normas electorales. Mientras que las sentencias del Tribunal solamente son vinculantes para las partes en una controversia, ${ }^{130}$ las mismas deberían constituirse en claras referencias para otros casos. No obstante, estas normas basadas en la identificación de una norma europea común, tiene que ser necesariamente amplia. ${ }^{131}$ De lo contrario, el TEDH será reprochado por haber "legislado", como fue el caso de Hirst vs. Reino Unido. De manera más general, la amplitud de las normas inevitablemente refleja el punto más complejo y difícil del TEDH como tribunal internacional, el cual debe involucrarse en asuntos tan delicados como los arreglos electorales de los Estados partes. El juez Levits llamó a esto "dilema" del TEDH y observó que, "por un lado, es tarea del TEDH proteger los derechos electorales de los individuos; pero, por el otro, señala que no debe sobrepasar los límites de su legitimidad explícita e implícita, ni tratar de gobernar en el marco constitucional

\footnotetext{
${ }^{128}$ Supra nota.

${ }^{129}$ Rusia había comenzado a oponerse a la OIDDH después de que la institución criticó duramente las elecciones parlamentarias de ese país en 2003. También los cuestionamientos de otro país parecen haber sido impulsados por las Ilamadas "revoluciones de color", Evers, supra nota, p. 235.

${ }^{130}$ Artículo 46, CEDH.

${ }^{131}$ Golubok's “Dos capas de protección”, distinguiendo entre la función del TEDH de salvaguardar el “núcleo” de los derechos electorales y un cuerpo más general de derecho blando desarrollado por otros órganos del Consejo de Europa, tales como la Comisión de Venecia, Golubok, supra nota, p. 390.
} 
Este obra forma parte del acervo de la Biblioteca Jurídica Virtual del Instituto de Investigaciones Jurídicas de la UNAM www.juridicas.unam.mx

DOl: http://dx.doil.org/10.22201/iij.24487910e.2015.7.10083

que los pueblos han creado para sí mismos". ${ }^{132}$ "Debido a que el TEDH, en principio, se ha limitado solamente a evaluar si se ha producido una violación, este "dilema" se ve agravado por el carácter vinculante y binario de sus decisiones."133

Por el contrario, la ventaja comparativa de las misiones de observación electoral es su forma más "suave" de establecer normas. La concreción de las normas electorales en los informes de las misiones se basa en un amplio conjunto de documentos "soft law" y en las mejores prácticas. Las amplias posibilidades de contextualización (por ejemplo, para explicar el incumplimiento de las normas electorales en el contexto de una situación específica de un país) ${ }^{134}$ y el carácter no vinculante de sus recomendaciones, parecen particularmente adecuadas para permitir la construcción de normas internacionales uniformes, en la diversidad de los sistemas electorales de los Estados.

En cuanto a la implementación de las normas electorales, factores como el retardo para dictar una decisión y los limitados medios con que cuenta el TEDH para dar apoyo a los Estados en la ejecución de una sentencia, obstaculizan la acción del TEDH. Por otro lado y en razón a que el TEDH se limita estrictamente a los fundamentos de la petición, sólo se trata el tema de la supuesta violación, en lugar de abordar de una manera más general la idoneidad del marco electoral de un país.

En cambio, los informes de la OIDDH evalúan la totalidad del proceso y ésta emite recomendaciones sobre los aspectos legales, políticos, electorales, así como otros aspectos relacionadas con la elección. Éstos proporcionan una base consistente para emprender un diálogo posterior a las elecciones con las autoridades pertinentes del Estado, pero también de otros actores, como partidos políticos, la sociedad civil, medios de comunicación o las mujeres candidatas. La flexibilidad de este modelo de cumplimiento basado en la cooperación, impulsa la aplicación de las normas electorales internacionales, toda vez que permite la diversidad en el nivel nacional. Por ello, parece más adecuado enfrentar las deficiencias de los procesos electorales multifacéticos, con

\footnotetext{
${ }^{132}$ Judge Levis, disidente de la opinión en Zdanoka (Chamber judgment), p. 17. Turkey, supra nota, p. 147; Harris, supra nota, pp. 724, 728 y ss.

${ }^{134}$ ODIHR Report on Uzbekistan, supra nota.
}

${ }^{133}$ Zdanoka vs. Latvia y Yumak and Sadak vs. Turkey, the ECtHR recurrió a un medio adicional: si bien no se estableció una violación, no obstante, señaló en su razonamiento, que las leyes vigentes no eran satisfactorias y en la necesidad de su enmienda; Zdanoka vs. Latvia, supra nota, p. 135; Yumak and Sadak vs. 
Este obra forma parte del acervo de la Biblioteca Jurídica Virtual del Instituto de Investigaciones Jurídicas de la UNAM www.juridicas.unam.mx

una variedad de actores. Dado su potencial, la OIDDH debería aumentar y ampliar sus actividades en el seguimiento de las misiones de observación. ${ }^{135}$

Por último, para mejorar aún más su legitimidad, ambas instituciones podrían considerar un aumento de la confianza mutua mediante las referencias cruzadas. Por ejemplo, la mención a las sentencias del TEDH, se pueden encontrar en los informes de las misiones de observación de las elecciones en el Reino Unido y Austria, con lo cual se mejoran las conclusiones de la OIDDH. ${ }^{136}$ Además, el TEDH podría, de ser posible, sustentar su razonamiento en la amplia documentación contenida en los informes de las misiones de observación electoral, ${ }^{137}$ lo que facilitaría el examen de los hechos. ${ }^{138}$ Dado el creciente número de casos sobre el derecho a elecciones libres presentadas al TEDH y la diversificación de los países a los que se despliegan misiones de observación y evaluación electoral de la OIDDH, que incluye también democracias consolidadas, parece que existe un amplio espacio para una fructífera interacción futura entre ambas instituciones.

\section{CONSIDERACIONES FINALES}

Entonces, ¿algo nuevo desde el fin de la guerra fría? Se ha argumentado en este estudio que "el derecho internacional ha penetrado el derecho interno" en cuanto a los procesos electorales en el período posterior a 1989 y ha comenzado a regir también en los asuntos nacionales, al menos en el nivel regional europeo.

Aun así, la valoración anterior muestra los desafíos de tal expansión del derecho internacional en un área tan delicada como los procesos electorales nacionales y los

${ }^{135}$ ODIHR Common Responsibility, supra nota.

${ }^{136}$ Frodl en OSCE-ODIHR EAM Final Report on Austria Presidential Elections, 25 de abril de 2010, 4 y la referencia a Hirst in the OSCE-ODIHR EAM Reporte final sobre Elecciones Generales en el Reino Unido, 6 de mayo de 2010, p. 6.

${ }^{137}$ De acuerdo al artículo 38 CEDH, el TEDH podrá participar en las investigaciones de oficio.

${ }^{138}$ El TEDH se basa considerablemente en los instrumentos jurídicos no vinculantes. Véase, Tanase vs Moldavia, donde el TEDH se refirió en su análisis a la reforma electoral de Moldavia de 2008, al Consejo de la Comisión europea contra el Racismo y la Intolerancia, a la Comisión de Venecia y al Consejo de reacciones de la Asamblea Parlamentaria de Europa frente a los cambios legislativos de Moldavia; Tanase vs. Moldova, supra nota, pp. 45 y ss. Véase Golubok sobre el papel de los instrumentos de derecho no vinculante en la jurisprudencia del TEDH, sobre el derecho a elecciones libres, Golubok, supra nota, pp. 386 y ss. 
Este obra forma parte del acervo de la Biblioteca Jurídica Virtual del Instituto de Investigaciones Jurídicas de la UNAM www.juridicas.unam.mx

órdenes constitucionales. La tensión inherente y la compleja tarea de dar cabida a las normas internacionales (unificadas) con la diversidad en el nivel nacional, en el corazón mismo del funcionamiento de un Estado, es particularmente evidente en materia electoral. Sobre todo ante la variedad de sistemas electorales, lo cual es una expresión muy directa de las condiciones históricas, culturales, legales, sociales y políticas de cada Estado. Por otro lado, hay que destacar que si bien la legitimidad procesal relacionada con el resultado, refuerza tanto al TEDH como el desarrollo de las misiones de observación electoral en la OIDDH, junto con la aplicación de las normas internacionales, estas dimensiones sólo pueden contribuir a aliviar la tensión existente.

Por lo que, las elecciones son una condición esencial para una democracia genuina, pero no suficiente. ${ }^{139}$ La gobernanza democrática es un concepto complejo y difícil, y necesita instituciones fuertes, participación y mecanismos de rendición de cuentas, incluyendo elementos como debates parlamentarios, justicia, Estado de derecho, derechos humanos, transparencia, acceso a la información y una administración pública responsable y eficaz. ${ }^{140}$ Una realización efectiva de las normas electorales es, fundamental, mas no suficiente para una verdadera gobernabilidad democrática. Las normas electorales aquí discutidas abordan la relación vertical entre los ciudadanos y sus gobiernos, y la posibilidad de que las ciudadanos de un Estado realicen, a través de elecciones, una rendición de cuentas de su gobierno. Un siguiente paso hacia la realización de la gobernanza democrática sería alcanzar una rendición de cuentas horizontal, que incluya la separación de poderes, la relación entre el Legislativo y el Ejecutivo, la independencia del Poder Judicial, o el control civil del sector de la seguridad.

139 O'Donnell, G., Cullellet, J.V et al. (eds.), The Quality of Democracy. Theory and Applications, 2004; Diamond, L. y Morlino, L., Assessing the Quality of Democracy, 2005. Ver también las resoluciones pertinentes de la Asamblea General de la ONU: ONU GA Res 55/96 sobre "la promoción y consolidación de la democracia", UN Doc. A / RES / 55/96, en la cual se exige a los Estados tomar medidas en una amplia gama de áreas, incluyendo los derechos humanos, los sistemas electorales, el Estado de Derecho o la participación de la sociedad civil, Pippan, C., supra nota, pp. 16 y 15.

${ }^{140}$ UNDEF, "Situating the UN Democracy Fund in the Global Arena. The Elements of Democracy", http://Www.un.org/democracyfund/XSituatingDemocracy.htm. De hecho, la encuesta de Freedom House 2010, llegó a la conclusión de que “...a pesar de los fraudes electorales y otras manipulaciones que se produjeron en varios países en 2009, el panorama mundial en los últimos cinco años sugiere que los gobiernos son más propensos a permitir elecciones relativamente honestas, que para permitir una prensa sin censura, una sociedad civil robusta, y un poder judicial independiente", Freedom House, supra nota, p.3. 
Este obra forma parte del acervo de la Biblioteca Jurídica Virtual del Instituto de Investigaciones Jurídicas de la UNAM www.juridicas.unam.mx

DOI: http://dx.doi.org/10.22201/iij.24487910e_2015.7.10083

Dada su complejidad, el desarrollo de normas internacionales en esta área se encuentra todavía en una fase embrionaria. ${ }^{141}$ No obstante, en vista de la creciente expansión del derecho internacional hacia el ámbito nacional en los últimos dos decenios, cabe preguntarse si la legitimidad de las normas internacionales sobre la rendición de cuentas horizontal de la gobernanza democrática, será el tema de un próximo artículo, tal vez en 2025 .

${ }^{141}$ Para una primera evaluación DRI, "Discussing International Standards for Democratic Governance. A Preliminary Research Repor", septiembre de 2007, http://Www.democracy-reporting.org/standards.htm/. En cuanto a las normas internacionales para las legislaturas democráticas, consulte NDI, "Hacia el Desarrollo de las Normas Internacionales de Legislaturas Democrática", enero de 2007. Véanse también proposiciones de la OSCE-OIDDH para los nuevos compromisos de la OSCE con los Estados participantes en lo que se refiere a los componentes necesarios de un gobierno democrático genuino, incluyendo separación de poderes, la clarificación del papel de la rama ejecutiva, la independencia del poder judicial y la transparencia legislativa y eficiencia, ODIHR Common Responsibility, supra nota. 